\title{
New Wireless Handheld Ultrasound-Guided Vacuum-Assisted Breast Biopsy (VABB) Devices: An Important Innovation in Breast Diagnosis
}

\author{
Silvia Bagnera ${ }^{1 *}$, Sebastiano Patania ${ }^{1}$, Luisella Milanesio ${ }^{2}$, Giovanni Gatti ${ }^{1}$, Renzo Orlassino ${ }^{3}$ \\ ${ }^{1}$ Department of Diagnostic Imaging and RT-Breast Unit, Local Health Unit (ASLTO4), Turin, Italy \\ ${ }^{2}$ Breast Screening Unit (Regional Reference Center), Regional Hospital “City of Health and Science of Turin”, Turin, Italy \\ ${ }^{3}$ Department of Patology, Local Health Unit (ASLTO4), Turin, Italy \\ Email: sbagnera@aslto4.piemonte.it
}

Received September 15, 2013; revised October 15, 2013; accepted October 22, 2013

Copyright (C) 2013 Silvia Bagnera et al. This is an open access article distributed under the Creative Commons Attribution License, which permits unrestricted use, distribution, and reproduction in any medium, provided the original work is properly cited. In accordance of the Creative Commons Attribution License all Copyrights (C) 2013 are reserved for SCIRP and the owner of the intellectual property Silvia Bagnera et al. All Copyright (C) 2013 are guarded by law and by SCIRP as a guardian.

\begin{abstract}
Vacuum-assisted breast biopsy (VABB) plays a fundamental role in the preoperative assessment of breast lesions providing large histology samples that are useful to define diagnoses and biological parameters to guide treatment planning. This technique has been used in our institute since 2000 and two new wireless handheld ultrasound-guided VABB devices have been introduced since May 2012. In this report we analyze our experience with these revolutionary devices which are able to provide the option of single-insertion contiguous tissue samples respectively with a 13/14-gauge aperture. Our initial experience on 75 lesions shows that these devices are safe, fast, procedurally advantageous for operators and well accepted by patients. Finally VABB procedures can markedly reduce the need for surgical biopsy.
\end{abstract}

Keywords: Breast Biopsy; Vacuum-Assisted Breast Biopsy; Ultrasound-Guided Histology Biopsy

\section{Introduction}

Mammography and ultrasound (US) frequently reveal suspicious nonpalpable lesions that require further workup to determine their true nature. This has led to the development of a number of noninvasive biopsy techniques in an attempt to reduce the need for diagnostic surgical biopsies [1]. They include fine needle aspiration cytology (FNAC) and percutaneous biopsy performed with an automated or semiautomated (Core Biopsy-CB Tru Cut, spring-loaded system) or a directional vacuum-assisted biopsy system (VABB).

In our institute, the latter method is used since 2000 in selected cases, e.g. microcalcifications (less than or equal to $1 \mathrm{~cm}$, with stereotactic guide) or lesions with initial needle aspirate findings classified as C1 or C3 according to international guidelines.

The transition from cytology to histology has been driven by the increased histological accuracy provided by larger tissue samples. In fact the possibility of false-

*Corresponding author. negative diagnoses is the most obvious shortcoming of core biopsy of the breast (CB-Tru Cut carries with it an overall false negative rate of $2.9 \%-10.9 \%$ ) [2].

The VABB devices offer consistent quality tissue samples and subsequent reduced possibility of false-negatives as well as underestimation of the disease process [3].

This report describes our initial experience with two new innovative wireless handheld US-guided VABB devices during the year 2012 and the first semester of 2013, and confirms the efficacy of these equipments with few complications during the procedure, with several procedural advantages for operators and with great benefit for women (less time, less trauma and less anxiety).

\section{Materials and Methods}

Over a 13 months period (May 3, 2012-June 17, 2013), 75 US-guided breast biopsies were performed at our institute: in 40 cases using the 13-gauge handheld VABB device Mammotome ${ }^{\circledR}$ Elite $^{\mathrm{TM}}$ (Devicor) and in 35 cases using the 14-gauge handheld VABB device Finesse ${ }^{\circledR}$ 
Ultra (BARD).

All the lesions were categorized using the Breast Imaging Reporting and Data System (BI-RADS) and all the biopsies were guided with an ultrasound scanner equipped with a 7.5 - $14 \mathrm{MHz}$ transducers (Esaote Biomedica AU5 Harmonic ultrasound scanner).

Both devices consist of a driver and a probe: the drivers have an ergonomic design and consist in a self-contained, handheld, reusable electro-mechanical vacuumassisted biopsy device that utilizes a rechargeable lithium-polymer battery (as shown in Figure 1(a)).

The single use probe is different in the two systems (as shown in Figures 1(b) and (c)).

The Mammotome ${ }^{\circledR}$ Elite $^{\mathrm{TM}}$ probe is a non-firing probe with a 13-gauge needle aperture: its 360-degree needle rotation allows you to adjust orientation of needle aperture based one position and approach to lesion. Its bladed probe tip moves easily through tissue while maintaining control of the needle and minimizing the time required between tissue samples (keeping the sampling chamber to the center of the target region).

In this kind of device the vacuum system (called Tru$\mathrm{Vac}^{\mathrm{TM}}$ ) both acquires and transports tissue from the biopsy site to the collection cup. This basket has also a special design with three pieces that consents a quick release of the cup with an easy tissue removal and enables touchless transfer of pathology.

The Finesse ${ }^{\circledR}$ Ultra probe is composed of a hollow outer cutting cannula (with a sharpened cutting edge) and an inner needle with a notched sample chamber and a sharpened trocar tip. During the sampling process, the driver creates a vacuum while simultaneously moving the outer cannula backward to expose the sample chamber. The tissue is drawn into the sample chamber; the outer cannula is released and moves forward over the sample chamber to cut the tissue. The tissue sample is transported via the sample chamber to the sample container, then the sample chamber is returned to the original position and is available to acquire another sample. Some lights indicate the operation status of the driver that also has an optional piercing mode able to retract the probe 20 - $22 \mathrm{~mm}$ to assist in penetrating dense breast tissue. Using imaging guidance, the probe's tip can be positioned to the appropriate depth: at the edge of the lesion, if the target must be pierced, or at the center of the target region (considering that the center of the sampling chamber is approximately $17 \mathrm{~mm}$ from the tip of the probe).

Both devices have an integrated coaxial cannula that may be detached after the biopsy and remains in the breast to retain a track to the biopsy site when placing a tissue marker (as CorMARK ${ }^{\mathrm{TM}} 14 \mathrm{G}$ in Mammotome ${ }^{\circledR}$ Elite $^{\mathrm{TM}}$ system and Gel Mark ${ }^{\circledR}$ UltraCor $^{\circledR} 14 \mathrm{G}$ in Finesse ${ }^{\circledR}$ Ultra system).

Both drivers automatically recognize their probe type;

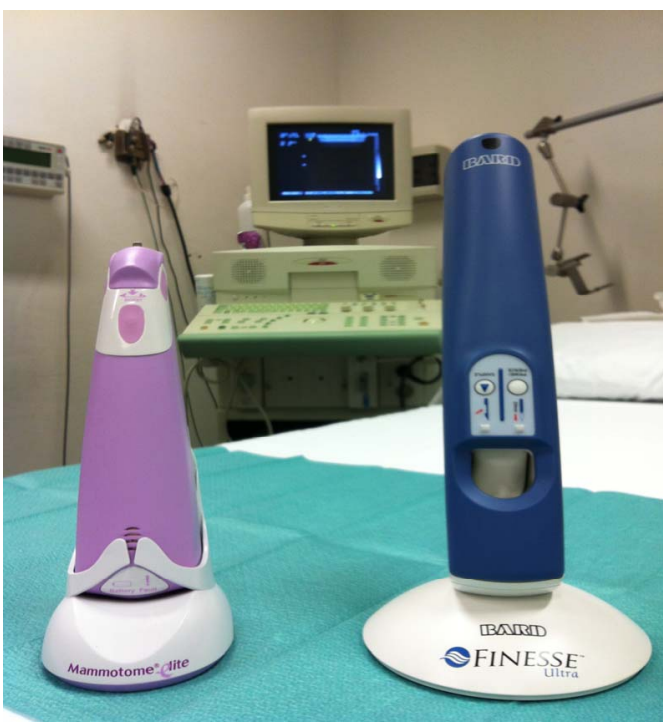

(a)

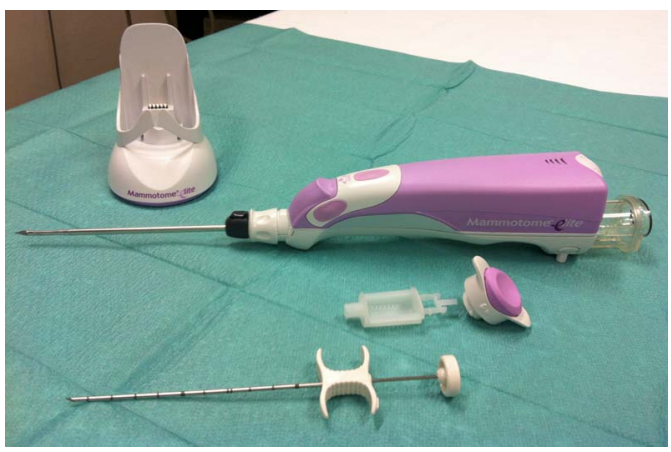

(b)

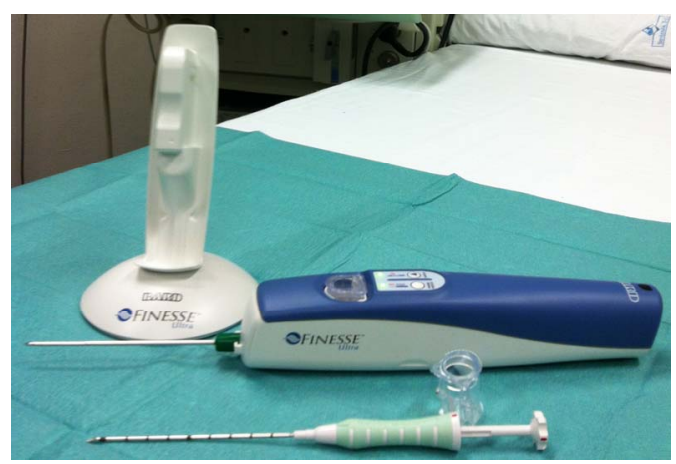

(c)

Figure 1. (a) Mammotome ${ }^{\circledR}$ Elite $^{\mathrm{TM}}$ and Finesse ${ }^{\circledR}$ Ultra Drivers. Both drivers have an ergonomic handheld design with a reusable electro-mechanical vacuum-assisted biopsy system; (b) Mammotome ${ }^{\circledR}$ Elite $^{\mathrm{TM}}$ Probe and Components. It is a nonfiring probe with a 13-gauge needle aperture and with an integrated coaxial cannula (as CorMARK ${ }^{\mathrm{TM}}$ 14gauge); its basket has a special design and consists of three pieces; (c) Finesse ${ }^{\circledR}$ Ultra Probe and Components. It has an ultra-sharp cutting tip (with a 14-gauge needle aperture and with an integrated coaxial cannula called UltraCor ${ }^{\circledR}$ 14-gauge) and an optional piercing mode (like a tru cut) able to penetrate into the dense breast tissue. It is also equipped with a lighting system of the basket. 
when the probe has been properly inserted, the driver performs an initialization sequence to synchronize the driver and probe. If the driver is left undisturbed after loading a probe (for 30 seconds in Mammotome ${ }^{\circledR}$ Elite $^{\mathrm{TM}}$ system or for 120 seconds in Finesse ${ }^{\circledR}$ Ultra system), the driver will enter a sleep mode to conserve battery charge.

All the 75 procedures included the following steps:

- Sonographic visualization of the lesion (with colorDoppler examination to identify blood vessels whose puncture might cause significant hematomas);

- Local anesthesia (obtained with buffered 1\% lidocaine injected into the skin and superficial tissues and with buffered $1 \%$ lidocaine containing epinephrine within the deeper breast tissues);

- US-guided placement of the handheld VABB probe at the level of the target lesion;

- Lesion sampling;

- Optional post-procedural placement of a localizing nonmagnetic titanium clip (followed by the execution of two orthogonal projections if necessary to confirm the exact location of the clip);

- Compression and dressing of the wound (the hemostasis was achieved with manual compression of at least ten minutes duration immediately following removal of the biopsy device).

The new shape of the tip needle has eliminated the skin incision with a scalpel blade, also in patients with extremely fibrous breast.

For each biopsy the number of samples, the execution time and the complications (e.g. significant bleeding, infection, hematoma, skin defect) were recorded.

The appearance of hematomas or other problems (as skin defect) were also monitored with an ultrasound control at ten days post-biopsy.

Moreover, the technical positive advantages identified by operators were evaluated. The subjective outcome of the procedure and women satisfaction were assessed according to a patient-completed questionnaire that incorporated a multistage scoring system like VAS (Visual Analogue Scale), proposed at the end of the procedure and a month after biopsy (with a phone interview).

According to the micro-histology outcome, our diagnostic algorithm provided the following actions: repeat VABB or surgery for lesions classified at the Breast Imaging Reporting and Data System (BI-RADS) as B1 (inadequate), follow-up based on radiological findings for lesions classified as B2 (benign); surgery for all other lesions (classified as B3-probably benign/B4-abnormality/B5-malignancy).

The final histology was compared with the micro-histology outcome obtained by biopsy with the newhandheld VABB devices, to determine whether there was an underestimation of disease.

Follow-up was indispensable to identify patients with $\mathrm{VABB}$ and/or other diagnostic findings that were false negatives: our experience suggested that follow-up at 6 12 months (depending on VABB and sonographic findings) was indicated to exclude false negative results.

\section{Results}

Most of the lesions biopsied were opacities on mammography (70.6\%); the others were areas of increased density. The mean age of the women was 58 years (range: 42 - 77 years).

All the lesions were evaluated previously with diagnostic breast US: they presented as a palpable node in the $74.6 \%(56 / 75)$ of cases and they were $8-30 \mathrm{~mm}$ in diameter, with an average lesion size of $16.5 \mathrm{~mm}$.

Using BI-RADS, the lesions were mammography/ultrasound classified: $42.6 \%$ as BI-RADS category R3U3 (nonspecific benign), 10.7\% as BI-RADS category R4U4 and $46.7 \%$ as BI-RADS category R5U5.

The $48 \%(36 / 75)$ of the patients had previously undergone fine needle aspiration cytology, which revealed 15 C1, 6 C2, 3 C3, 6 C4, 6 C5. In the remaining patients the initial diagnostic work-up had been based on histology (Table 1).

Table 1. FNAC and VABB micro-histology outcomes.

\begin{tabular}{|c|c|c|c|}
\hline & $\begin{array}{c}\text { BI-RADS } \\
\text { Classification on }\end{array}$ & \multicolumn{2}{|c|}{ Outcomes } \\
\hline \multirow{9}{*}{$\begin{array}{l}40 \text { Mammotome }^{\circledR} \\
\text { Elite }^{\mathrm{TM}} \text { procedures }\end{array}$} & \multirow{4}{*}{16 R3U3 } & FNAC & VABB \\
\hline & & $2 \mathrm{C} 1$ & $2 \mathrm{~B} 2$ \\
\hline & & $3 \mathrm{C} 2$ & $3 \mathrm{~B} 2$ \\
\hline & & --- & 9 B2 \\
\hline & \multirow{3}{*}{6 R4U4 } & $2 \mathrm{C} 3$ & 2 B3 \\
\hline & & $\begin{array}{l}4 \mathrm{C} 4 \\
2 \mathrm{C} 1\end{array}$ & $\begin{array}{l}4 \mathrm{~B} 5 \\
2 \mathrm{~B} 5\end{array}$ \\
\hline & & $3 \mathrm{C} 5$ & 3 B1 \\
\hline & \multirow{4}{*}{18 R5U5 } & $1 \mathrm{C} 5$ & $1 \mathrm{~B} 5$ \\
\hline & & $6 \mathrm{C} 1$ & $6 \mathrm{~B} 5$ \\
\hline \multirow{10}{*}{$\begin{array}{l}35 \text { Finesse }^{\circledR} \text { Ultra } \\
\text { procedures }\end{array}$} & & ---- & 8 B5 \\
\hline & & 4 C 1 & $4 \mathrm{~B} 2$ \\
\hline & \multirow{2}{*}{16 R3U3 } & $3 \mathrm{C} 2$ & $3 \mathrm{~B} 2$ \\
\hline & & ---- & 8 B2 \\
\hline & \multirow{3}{*}{2 R4U4 } & $1 \mathrm{C} 3$ & $1 \mathrm{~B} 3$ \\
\hline & & $2 \mathrm{C} 4$ & 2 B5 \\
\hline & & $1 \mathrm{C} 5$ & $1 \mathrm{~B} 1$ \\
\hline & \multirow{3}{*}{17 R5U5 } & $1 \mathrm{C} 5$ & $1 \mathrm{~B} 5$ \\
\hline & & $1 \mathrm{C} 1$ & $1 \mathrm{~B} 5$ \\
\hline & & -.-- & 14 B5 \\
\hline
\end{tabular}

FNAC: fine needle aspiration cytology; VABB: vacuum-assisted breast biopsy. 
Using the two new handheld VABB devices, needle multiple contiguous tissue samples of single-insertion were obtained (as shown in Figures 2(a)-(d)) and the mean number of specimens collected per patient was 6 (range: 2 - 10). Then they were submitted for histopathologic evaluation.

The number of samples was dependent on lesion size and on US conspicuity during the procedure: in some cases the procedure was early aborted because of a hematoma obscured the lesion and so only two specimens were obtained. These four cases complicated by bleeding during VABB procedure were considered inadequate (B1) but because they had positive cytology for cancer (C5), they were sent to surgery.
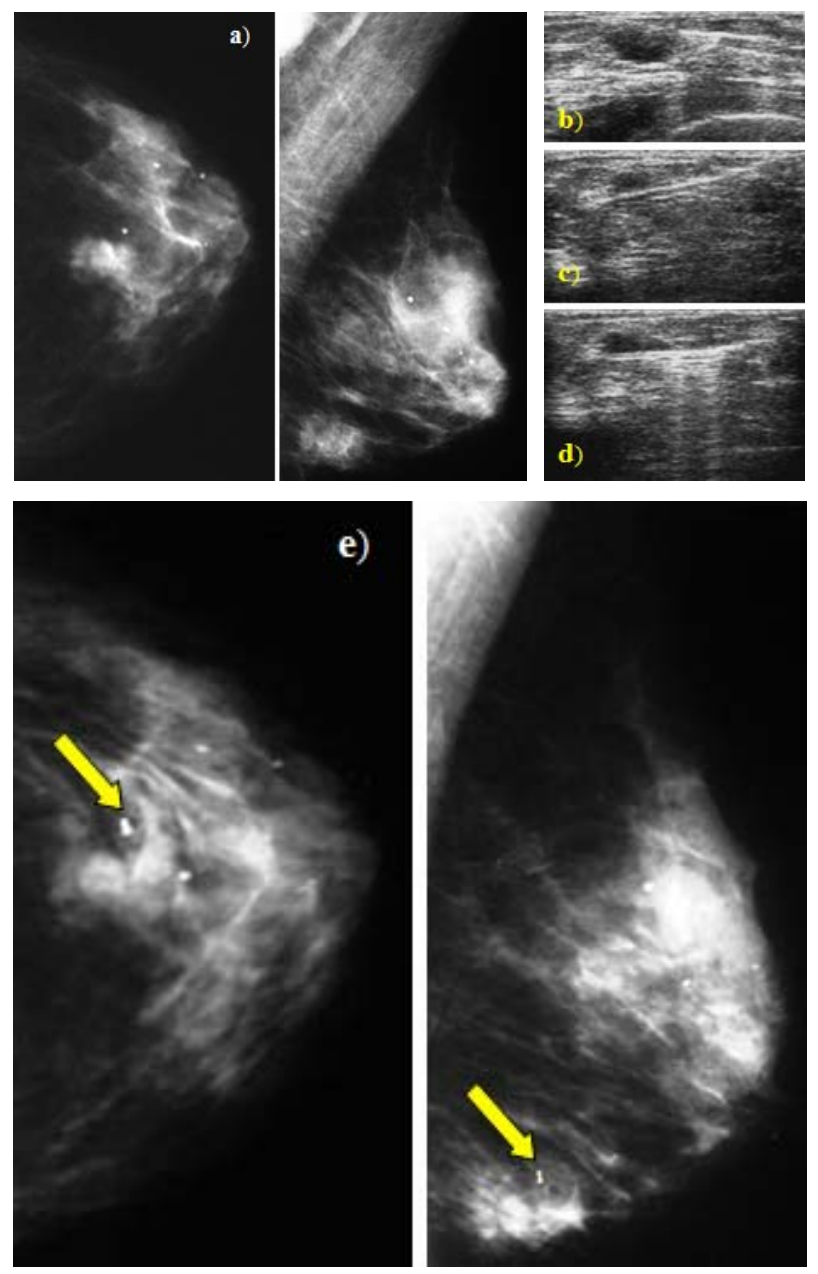

Figure 2. (a)-(d) Example of US-guided VABB procedure using an handheld single-insertion device. Case of mammographic opacity in Q3 - Q4 of the left breast (a) corresponding to an hypoechoic palpable formation at the US (b) subjected to multiple sampling by single insertion ((c), (d)); (e) Example of clip positioning at the end of VABB procedure. After tissue sampling was completed, a marking nonmagnetic titanium clip was positioned and the localization was confirmed with two orthogonal projections postprocedure (as shown by arrow).
In our cases no lesion was completely removed.

After tissue sampling was completed, a marking nonmagnetic titanium clip was positioned in seven cases of nonpalpable lesions (9\%: 7/75) (as shown in Figure 2(e)).

With both devices, the entire VABB procedure ended in 5 to 8 minutes because the time to acquire tissue was extremely fast and precise.

Few minor complications were observed: only bleeding during some procedures.

No patient experienced a vasovagal response (this confirmed that the supine patient position used during USguided procedures virtually eliminates the risk of substantial patient injury from a vasovagal episode).

None skin defects were seen and no residue of hematoma was noted at US 10 days later.

All the lesions classified B1-C5 were confirmed malignant at the surgical histology findings (two of those were infiltrating lobular carcinomas, CLI and two were an infiltrating ductal carcinomas, CDI).

The three lesions with a micro-histology outcome as BIRADS category 3 (B3) were noted at the surgical histology findings to be atypical ductal hyperplasias.

All the 39 lesions with micro-histology outcome as BI-RADS category B5 were malignant at the surgical outcome, of those: 27 were infiltrating ductal carcinoma (CDI), 6 were infiltrating lobular carcinoma (CLI), 4 were mixed infiltrating carcinoma (ductal + lobular) and the remaining 2 lesion were in situ ductal carcinoma (CDIS) (Table 2).

In our study, the sensibility was $87.5 \%$ for Mammotome $^{\circledR}$ Elite $^{\mathrm{TM}}$ and $94.7 \%$ for Finesse ${ }^{\circledR}$ Ultra and the specificity was $100 \%$ for both devices.

The technical elements reported as positive by the operators were: the ergonomics of the handpiece, light weight (with less arm fatigue), handling to 360 degrees of the needle, the speed of execution.

The pain perceived by women during the procedure was found to be scarce or tolerable in $93 \%$ of cases and the patients were satisfied of the procedure (in $97 \%$ of cases), without significant differences between the opinions expressed immediately after the procedure and the statements released on the phone a month later.

During the follow-up examination (6 - 12 months later), the site of the biopsy and/or the remaining lesional tissue was identified (with or without the aid of the clip) without VABB interferences on the subsequent mammographic or sonographic assessments of the breast.

Thus far our follow-up has not revealed any cases of false negativity.

\section{Discussion}

The intention of this report is show our early experience with two new wireless handheld US-guided VABB de- 
Table 2. VABB micro-histology and surgical histology outcomes.

\begin{tabular}{|c|c|c|c|}
\hline & & VABB microhistology & Surgery histology \\
\hline & & 14 В2 а. & ------------ \\
\hline & 16 R3U3 & & \\
\hline & & 2 B3 & 2 Atypical ductal hyperplasias \\
\hline \multirow[t]{5}{*}{ Mammotome ${ }^{\circledR}$ Elite $^{\mathrm{TM}}$ procedures } & $6 \mathrm{R} 4 \mathrm{U} 4$ & 6 B5 & 4 Mixed infitrating carcinoma (ductal + lobular) 2 CDI \\
\hline & & 3 B1 & 1 CLI, 2 CDIS \\
\hline & 18 R5U5 & 15 B5 & 15 CDI \\
\hline & & $15 \mathrm{~B} 2 \mathrm{a}$. & ----------- \\
\hline & $16 \mathrm{R} 3 \mathrm{U} 3$ & $1 \mathrm{~B} 3$ & 1 Atypical ductal hyperplasias \\
\hline \multirow[t]{3}{*}{ Finesse ${ }^{\circledR}$ Ultra procedures } & 2 R4U4 & 2 B5 & 2 Mixed infiltrating carcinoma (ductal + lobular) \\
\hline & & $1 \mathrm{~B} 1$ & $1 \mathrm{CLI}$ \\
\hline & 年 & $16 \mathrm{~B} 5$ & $16 \mathrm{CDI}$ \\
\hline
\end{tabular}

a. Fibroadenomas and other nonspecific benign abnormalities. CDI = infiltrating ductal carcinoma; CLI = infiltrating lobular carcinoma; CDIS = in situ ductal carcinoma. The 38.6\% (29/75) of the lesions subjected to VABB procedures were B2 (benign) i.e. fibroadenomas and other benign non-specific abnormalities (such as stromal fibrosis or sclerosing adenosis); the lesions resulted B3 (probably benign) at micro-histology outcome were atypical ductal hyperplasias at the surgical histology and all the lesions resulted B5 were confirmed as cancer. Four cases complicated by bleeding during VABB procedure were considered inadequate (B1) and resulted malignant (as indicated by the previous cytological outcome).

vices (respectively $13 \mathrm{G}$ and $14 \mathrm{G}$ ) though we recognize that our study is limited by the few cases and by the short interval of follow-up.

The ultrasound-guided FNAC is known to be a simple and an economical procedure which in expert hands can furnish good results, nonetheless when it yields non-diagnostic or ambiguous results (as category C3), other procedures are indicated for continuation of the diagnostic work-up. Moreover, malignant lesions (C4 and C5) may also be candidates for pre-surgical histology when it is important to know the hormone receptor status of a tumor before surgery (in case of neoadjuvant chemotherapy).

In our practice, we have transitioned from FNA/CB to VABB devices in an effort to obtain diagnostic accuracy for suspicious breast lesions, thereby minimizing and/or eliminating insufficient or inconclusive diagnosis. In fact multiple passages are often needed with CB-procedure to obtain adequate tissue samples (because frustules are often small and fragmented), increasing the patient's discomfort and the risk of complications in particular with lesions located near sensitive areas (e.g. near a breast implant, near the patient's chest wall or in the axilla).

In our opinion, US-guided VABB procedure with both of these new devices has certain features that make them superior to CB-Tru Cut.

They include:

- less risks of sampling errors thanks to take multiple contiguous samples in a single insertion both for small or deep lesions, for complex cystic masses (with the possibility for pathologist to evaluate both solid component and fluid component) and for large masses (malignant and benign);

- a quick and easy repositioning of the needle within the mass with the needle aperture that remains closed after each sample;

- a sampling chamber that is highly visible during sampling and can perform removal of small masses (as an excellent alternative to surgical excisional biopsy in presence of fibroadenomas);

- a clear visualization of the tissue sample into the collection cup and easy transport to pathology thanks to the closed vacuum system that transports tissue from the biopsy site to the cup ;

- a time significantly short to acquire quality tissue samples;

- a minimal learning curve needed for physicians (thanks to the ergonomic features and to the ease-ofuse) and the absence of additional staff or space;

- an accurate placement of tissue markers, facilitated by the integrated coaxial cannula.

These exciting innovative devices have greatly simplified the overall procedure by eliminating the need to remove the biopsy needle from breast tissue in order to retrieve a tissue sample: in fact there is no need to revisualize the mass or to prepare a needle with multiple hand maneuvers.

Other additional advantages of US-guided VABB are: the nonappearance of mammographic alterations in subsequent studies or scars that are difficult to interpret, the appreciation of this new tool by women and the virtually 
absence of complications.

\section{Conclusion}

We believe that the wireless handheld single-insertion US-guided VABB devices reduce the possibility of falsenegatives and the underestimation of disease.

In our experience, these two new devices are effective in the characterization of breast lesions and they complete the tools at our disposal resulting in rapidity, manageability and easy transportability; in particular, they have different procedural advantages for the operators and the single insertion is a great comfort for women.

\section{REFERENCES}

[1] F. Abbate, E. Cassano, S. Menna and G. Viale, "Ultrasound-Guided Vacuum-Assisted Breast Biopsy: Use at the European Institute of Oncology in 2010,” Journal of Ultrasound, Vol. 14, No. 4, 2011, pp. 177-181.

[2] S. H. Parker, et al., "Sonographically Guided Directional Vacuum-Assisted Breast Biopsy Using a Handheld Device,” AJR, Vol. 177, No. 2, 2001, pp. 405-408.

[3] J. R. Simon, et al., "Accuracy and Complication Rates of US-guided Vacuum-assisted Core Breast Biopsy: Initial Results,” Radiology, Vol. 215, No. 3, 2000, pp. 694-697. http://dx.doi.org/10.1148/radiology.215.3.r00jn37694 